Jens Vertongen* and Derek Kamper

\title{
Design of a 3D printed hybrid mechanical structure for a hand exoskeleton
}

https://doi.org/10.1515/cdbme-2020-2003

\begin{abstract}
Stroke survivors often have difficulty performing activities of daily living (ADLs) due to hand impairments. Several assistive devices have been developed for stroke survivors to assist them with ADLs but most of these devices are difficult to don and doff for a stroke survivor due to highly flexed postures of the wrist and digits. This paper presents a hybrid 3D printed mechanical structure for an assistive hand exoskeleton created for stroke survivors. The design facilitates donning and doffing of the assistive exoskeleton by enabling an approach entirely from the dorsal side of the hand, thereby allowing the fingers to stay flexed. The design criteria, resulting design and the prototype development are presented. The initial prototype of the structure, using a hybrid combination of rigid and flexible materials, was lightweight (only $185 \mathrm{~g}$ ), while maintaining a high range of motion. Future directions for further improvements and user studies are described.
\end{abstract}

Keywords: assistive technologies; exoskeleton; hand; orthosis; rehabilitation; stroke.

\section{Introduction}

Stroke often results in a severe impairment of the upper limb, particularly the distal segment, in stroke survivors. In 2010 there were 6.7 million stroke survivors in the United States alone, with 795,000 new or recurring strokes occurring each year [1]. The symptoms of this impairment include paresis, involuntary muscle contraction patterns and impaired movements of the paretic hand [2]. These can make it difficult for stroke survivors to perform activities of daily living (ADLs) such as eating, bathing and dressing. Therefore, an assistive device for the distal upper limb that

\footnotetext{
${ }^{*}$ Corresponding author: Jens Vertongen: Department of BioMechanical Engineering, Delft University of Technology, Delft, The Netherlands, E-mail: vertongen.jens@gmail.com

Derek Kamper, Joint Department of Biomedical Engineering, University of North Carolina at Chapel Hill and North Carolina State University, Raleigh, USA
}

can assist with these activities has the potential to improve the stroke survivor's quality of life.

Several assistive devices have been reported in literature. These devices commonly use a rigid structure [3-5] or a glove that routes tendons along the fingers [6-8]. These devices are often difficult to don and doff due to the typically flexed hand posture and the lack of voluntary finger extension. Coupling an assistive device to a stroke survivor's hand can be especially challenging, due to the limited available contact area on the digits and the substantial resistance to even passive extension of the digits that arises from involuntary muscle activation [9].

In this paper we present the design of a hybrid, 3D printed mechanical structure of a hand exoskeleton intended to actuate the fingers of stroke survivors. The aim is to improve the donning process while providing the capability of both flexion and extension assistance from a single actuator for each finger located on the dorsal side of the hand. The actuator drives push-pull cables that can either flex or extend the joints of the digit. Thus, the mechanical structure, composed of rigid and flexible materials, must serve as the interface between the actuator and the digit.

\section{Design requirements}

The exoskeleton structure must fulfill multiple roles: guiding the cables actuating the hand, transmitting moments to the fingers and thumb, and preventing excessive joint rotation. The design requirements are listed in the following three categories: structural, safety and transmission requirements.

\section{Structural requirements}

The device has to fit the hand of the user while maintaining a low profile (maximum height of $25 \mathrm{~mm}$ above the finger) and should be easy to don and doff. The structure has to guide the push-pull cables and should keep the palmar surface free and accessible to minimize interference with the user's sensory feedback and manipulation of objects. The device has to be lightweight with a mass under $300 \mathrm{~g}$. 
The range of motion (ROM) of the hand with the device should be close to the normal ROM: 100,105 and $85^{\circ}$ for the MCP, PIP and DIP respectively and 56 (MCP) and $73^{\circ}$ (IP) for the thumb [10]. Furthermore, the fingers should be able to abduct.

\section{Safety requirements}

The mechanical structure should prevent excessive joint flexion and extension. Moving parts have to be shielded to prevent tissue or body parts to be pinched.

\section{Transmission requirements}

Ultimately, the structure has to transmit the moments from the cables to the finger joints. The friction between the cables and the guides should be as low as possible.

\section{Exoskeleton structure design}

\section{Full structure}

The structure was designed through an iterative process of 3D design, rapid prototyping and verification. Drawing on hand dimensions from the literature [11], we designed a prototype using 3D CAD software (Figure 1). The mechanical structure is a hybrid design of rigid components, attached to the digit segments that guide the cables and prevent excessive joint rotation, and a flexible inner lining that connects the rigid components and improves comfort and functionality. The exoskeleton can be donned entirely from the dorsal side of the hand, with the rigid pieces deforming to accommodate the finger, thereby generating a clamping force against the sides of the finger. The structure extends to the forearm in order to provide bracing to maintain the wrist in a functional extended posture. This splint structure also provides housing for the actuators and electronics. Straps around the hand and wrist help to secure the location of the device on the body.

\section{Finger structure}

The anatomical differences in the distal phalanx (DP) of the thumb and the pinky finger, in comparison with the index, middle and ring fingers, require a different form factor for these segments (see Figure 1). We employed conical shapes
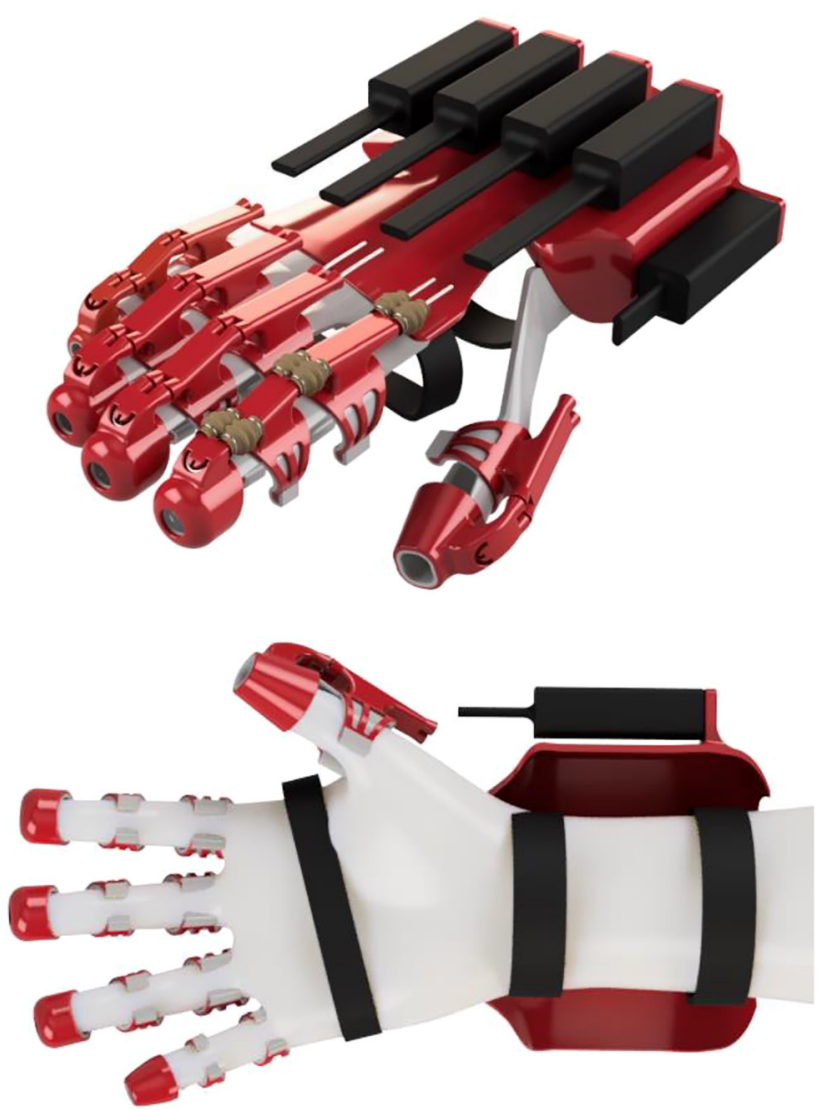

Figure 1: Full overview of the mechanical structure in red with the flexible lining in gray and actuators in black. Top: dorsal view. Bottom: palmar view.

for the components of the intermediate phalanx (IP) to ensure proper fitting on the finger segment.

Adjacent cable-guide segments meet during joint extension to prevent hyperextension of the joints (Figure 2). To prevent hyperflexion of the joints, possible pinching and buckling of the cable, we attached a fabric sleeve at the ends of the cable guides around the wire. These fabrics clamp over the conical end and are secured with a metal ring (Figure 3 ).

The cable guides on the dorsal side of the fingers are easily customizable to achieve different moment arms around the joints. We used two cables for a better lateral
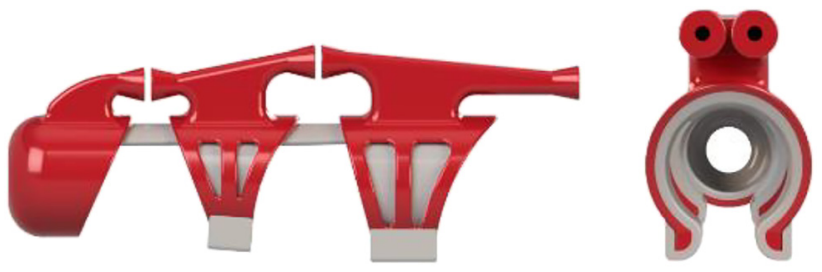

Figure 2: The structure of one finger. 


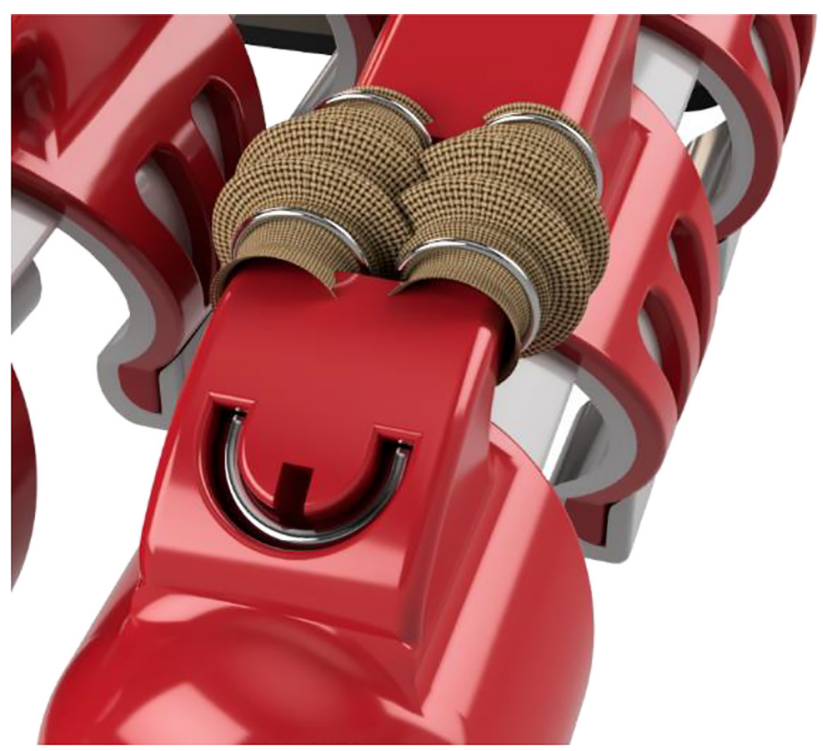

Figure 3: Detail of the sleeves preventing cable buckling.

stability to actuate the fingers. The friction of the cable, governed by the capstan Equation (1), should be minimized [12].

$$
T_{\text {out }}=e^{-\mu \phi} T_{\text {in }}
$$

In Equation (1), $\mu$ is the friction coefficient and $\phi$ is the deflection angle of the cable segment that is sliding in the tube. We designed the path inside the rigid component with the smallest curvature possible in order to minimize friction along the cable. Figure 4 shows a section of the finger structure where the cable path is visible.

The push-pull cable locks in the front of the structure in a groove above the DP (see Figure 3). This groove facilitates the assembly of the cable in the structure. The recess in this groove permits easy removal of the cable with a screwdriver.

\section{Arm structure}

The arm structure supports the actuators, transmission and electronics, in addition to maintaining a neutral wrist

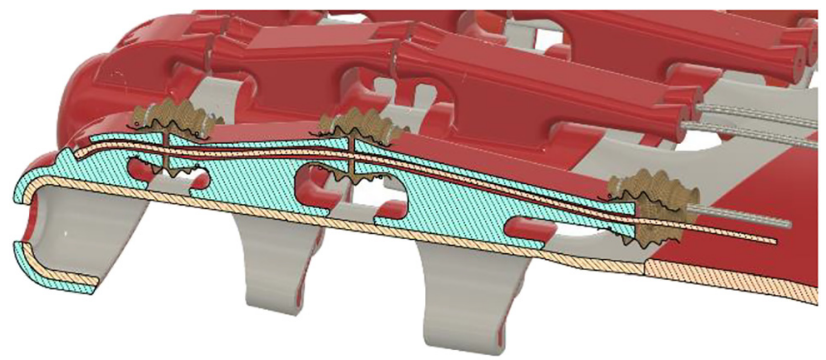

Figure 4: Section of the index finger showing the path of the cable within the guide. posture (see Figure 5). Velcro straps around the hand, wrist and forearm secure the location on the arm. We placed the actuators locally, on the forearm, to avoid frictional losses inherent to the use of long Bowden cables needed with remotely placed actuators.

\section{Prototype development}

The final prototype, realized with acrylonitrile butadiene styrene (ABS) and thermoplastic polyurethane (TPU) through 3D printing, proved to be lightweight and comfortable. The mass of the entire mechanical structure was only $185 \mathrm{~g}$. The distal portion that actually moves with the fingers and thumb was $82 \mathrm{~g}$ in total, or an average of roughly $16 \mathrm{~g}$ per digit; the forearm brace accounts for the other $103 \mathrm{~g}$. The ROM of the fingers, while wearing the structure when not actuated, is very close to the normal ROM (see Table 1). The finger structures in black and the arm structure in white are shown in Figure 6.

\section{D printing and material selection}

We used 3D printing as a rapid prototyping method to produce the exoskeleton structure due to the various benefits such as easily available, quick and the ability to produce complex shapes.

The structure was produced with two different 3D printers. The rigid and flexible material of the finger structures was produced by the BCND Sigma R17 3D printer. The rigid material of the arm structure was printed

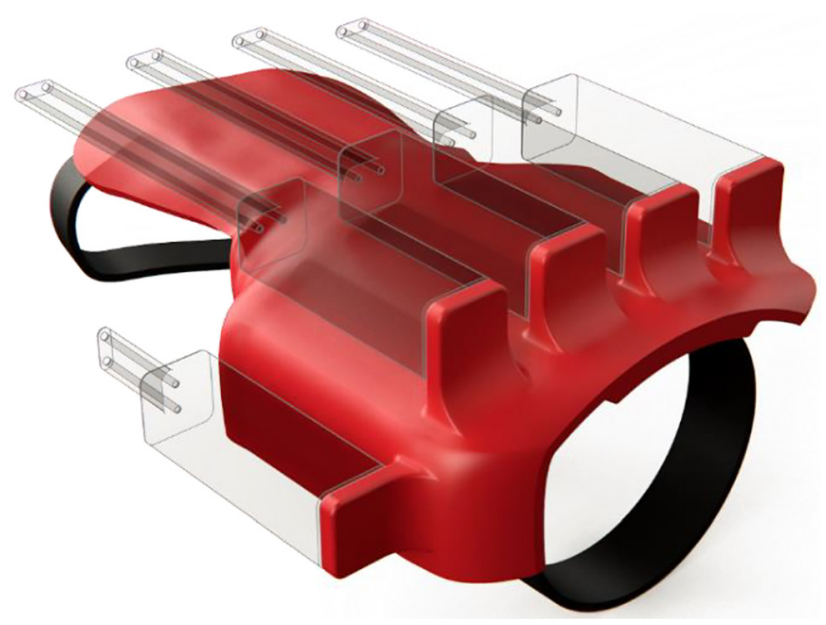

Figure 5: The arm structure that supports the actuators. Motors and cables are represented with transparent bodies. 
Table 1: Weight and ROM of the prototype.

\begin{tabular}{lrrr}
\hline Weight & $\begin{array}{r}\text { Fingers } \\
\mathbf{8 2 ~} \mathbf{~ g}\end{array}$ & $\begin{array}{r}\text { Arm part } \\
\mathbf{1 0 3} \mathbf{~ g}\end{array}$ & $\begin{array}{r}\text { Total } \\
\mathbf{1 8 5} \mathbf{~ g}\end{array}$ \\
\hline ROM & MCP ( $\left(^{\circ}\right.$ & PIP ( $\left(^{\circ}\right.$ & DIP ( $)$ \\
\hline Thumb & 50 & - & 70 \\
Index finger & 80 & 85 & 75 \\
Middle finger & 85 & 95 & 75 \\
Ring finger & 90 & 95 & 75 \\
Pinky finger & 75 & 85 & 75 \\
& & & \\
\hline Normal ROM thumb & 56 & - & 73 \\
Normal ROM finger & 100 & 105 & 85 \\
\hline
\end{tabular}

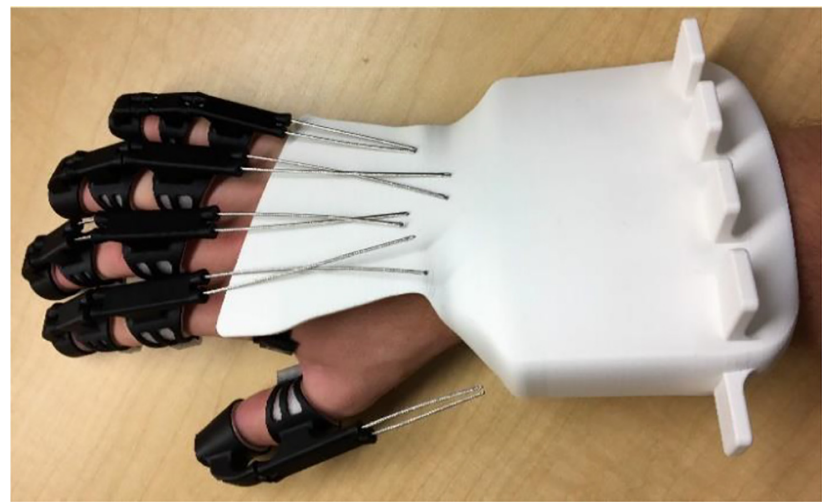

Figure 6: The final prototype.

by the Stratasys Dimension 1200 3D printer that has a larger build volume to produce the arm structure.

We employed ABS to produce the rigid components. This material was selected rather than polylactic acid (PLA) due to its greater toughness. A more flexible material, TPU, was chosen for the liner.

\section{Performance}

The weight and the range of motion of the exoskeleton are important for the functionality and comfort for the user. Table 1 lists the weight and the range of motion of the exoskeleton. The normal ROM values of the thumb and the fingers [10] are shown for reference. The exoskeleton proved to be comfortable to wear for extended periods of time. This was tested for a period of $1 \mathrm{~h}$ of use by one of the authors while performing ADLs. No pain or discomfort was reported for the duration of the test. Due to the large range of motion and slim profile, the author was able to grasp most everyday objects. There was some minor discomfort in pronation and supination, however, due to the location of the structure on the forearm.

\section{Discussion}

An actuated hand exoskeleton has the potential to improve the quality of life for stroke survivors by assisting with ADLs. The lightweight mechanical structure developed in this study helps to make such a device possible. Easily customizable to the shape of the user's hand, the structure can accommodate the push-pull cables used to drive the fingers while preventing joint hyperextension. The ability to put on the device entirely from the dorsal side of the hand greatly improves donning for stroke survivors with a flexed resting posture and resistance to passive extension. The slim profile, high range of motion and low mass result in a functional and comfortable structure.

The major limitation of the current structure is the TPU used for the flexible liner. A material with greater elasticity could be better suited for the application and could improve user comfort. Further development of the design and user testing are needed to improve and validate the design.

Research funding: Funded by the United States Department of Health and Human Services, National Institute on Disability, Independent Living, and Rehabilitation Research (NIDILRR), Rehabilitation Engineering Research Center (RERC) program funding mechanism: Collaborative Machines Enhancing Therapies (COMET), Grant number 90REGE0005-01-00.

Author contributions: All authors have accepted responsibility for the entire content of this manuscript and approved its submission.

Conflict of interest: Authors state no conflict of interest. Informed consent: Informed consent was obtained from all individuals included in this study.

Ethical approval: The local Institutional Review Board deemed the study exempt from review.

\section{References}

1. Go AS, Mozaffarian D, Roger VL, Benjamin EJ, Berry JD, Borden WB, et al Executive summary: heart disease and stroke statistics-2013 update: a report from the American heart association. Circulation 2013;127:143-52.

2. Gresham GE, Stason WB, Duncan PW. Post-stroke rehabilitation. Darby, PA: Diane Publishing Company; 2004. 
3. Cempini M, Cortese M, Vitiello N. A powered finger-thumb wearable hand exoskeleton with self-aligning joint axes. IEEE/ ASME Trans Mechatron 2015;20:705-16.

4. Ho NSK, Tong KY, Hu XL, Fung KL, Wei XJ, Rong W. An EMG-driven exoskeleton hand robotic training device on chronic stroke subjects: task training system for stroke rehabilitation. In: IEEE international conference on rehabilitation robotics. IEEE, New York City; 2011, 1-5.

5. Chiri A, Giovancchini F, Vitiello N, Cattin E, Roccella S, Vecchi F, et al HANDEXOS: towards an exoskeleton device for the rehabilitation of the hand. In: IEEE/RSJ international conference on intelligent robots and systems. IEEE, New York City; 2009, 1106-11.

6. In H, Kang BB, Sin M, Cho KJ. Exo-glove: a wearable robot for the hand with a soft tendon routing system. IEEE Robot Autom Mag 2015;22:97-105.

7. Nycz CJ, Delph MA, Fischer GS. Modeling and design of a tendon actuated soft robotic exoskeleton for hemiparetic upper limb rehabilitation. In: 37th Annual international conference of the IEEE engineering in medicine and biology society (EMBC). IEEE, New York City; 2015, 3889-92.

8. Rose CG, O'Malley MK. Hybrid rigid-soft hand exoskeleton to assist functional dexterity. IEEE Robot Autom Lett 2019;4:73-80.

9. Kamper DG, Rymer WZ. Quantitative features of the stretch response of extrinsic finger muscles in hemiparetic stroke. Muscle Nerve 2000;23:954-61.

10. Hume M, Gellman H, McKellop H, Brumfield RH. Functional range of motion of the joints of the hand. J Hand Surg 1990;15:240-3.

11. Buryanov A, Kotiuk V. Proportions of hand segments. Int J Morphol 2010;28:755-8.

12. Kaneko M, Yamashita T, Tanie K. Basic considerations on transmission characteristics for tendon drive robots. In: Robots in unstructured environments: IEEE, New York City; 1991, 827-32. 